\title{
Stock Portfolio Analysis Using Markowitz Model
}

\author{
Indah Nur Safitri ${ }^{a}$, Sudradjat $^{a}$, Eman Lesmana $^{a}$ \\ ${ }^{a}$ Department of Mathematics, Faculty of Mathematics and Natural Sciences, Padjadjaran University, \\ INDONESIA.
}

*Corresponding author: indnursafit16@gmail.com

\begin{abstract}
A common problem that often occurs in investment is the selection of the optimal portfolio according to the wishes of investors. This thesis ueds the Markowitz Model as a basis to formed a model to choose the optimal portfolio that provided the lowest risk. Efforts to minimize risk were carried out by conducting a diversification strategy. After the selection of several companies with the criteria of capitalization value and DER (Debt Equity Ratio), a combination of stocks is formed to form a portfolio. The formed portfolio was then analyzed to determine the optimal proportion of each stock. Using the Markowitz model, which is then solved by Non Linear Programming, an optimal portfolio is obtained with the proportion of each stock minimizing risk. In general, the results of this analysis indicate that portfolios with more stocks will produce lower risks compared to portfolios with fewer stocks, thus providing optimal diversification solutions, namely portfolios with members of five stocks with optimal risk of $0.886 \%$.
\end{abstract}

Keywords: risks, Stock Portfolio, Markowitz Model, Lagrange

\section{Introduction}

Over the past years, investing interest increases with the advancement of information and communication technology. Technological advancements simplify the process of transactions in investment, both in the process of buying, selling and overseeing the value of investments. According to Tandelilin (2010) investment is a form of investment both directly and indirectly, short term and long term, with expectations of benefits from its investment. Investments can be made individually or within organizations. The main purpose of investing is to optimizing the tevel of returns with the lowest risk (Kalfin et al, 2019).

One of the most popular investment products is stocks (Kalfin et al, 2019). Stocks itself are proof of investor ownership of a company. Stocks as an investment product can certainly provide the 
highest profits, but it can also provide a large risk to investors (Tandelilin, 2010). Through the concept of diversification (the process of allocating funds to various stock investment alternatives) the risk or loss of a stock can be covered with profits on other stocks (Husnan, 2005). Through the process of stock diversification, investors need to conduct portfolio analysis. The analysis will help investors in making decisions to determine the most efficient or optimal portfolio that has the optimal profit rate or the least risky one. The model for portfolio analysis that considers the relationship of return and risk is the Markowitz model.

The portfolio analysis process could be done by using Markowitz model. Through the concept of minimizing risk or maximizing the rate of return, the model is widely used to analyze the optimization of a portfolio or as a basis for forming a new portfolio. The design of the stock portfolio using the Markowitz model was conducted by Cohen and Pogue (1967) to evaluate the work of a number of portfolios.

\section{Description of Problem}

This study discusses the determination of the optimal portfolio of a combination of five selected companies on IDX LQ 45, where the data used is historical stock closing price data for the period January 2014 - July 2018. In this study a Portfolio Optimization Model will be formed which will be completed with the Non-Linear Programming method with the aim minimize variants. The formation of an optimization model will then give the proportion of each share in each portfolio. Stockholders are assumed to have limited investment funds, amounting to 1 billion rupiah with the expectation of a portfolio return of $5 \%$. The funds will then be invested in the amount calculated by the proportion obtained from the optimal stock portfolio.

The selecting process of companies was done by choosing five blue chip company that listed in IDX LQ 45 with the higher rate of Market Capitalization (Market Cap.) and the lower rate DER. The Market Cap. is the total market value of all of a company's outstanding shares. Market Cap. shows the value of the business of one company which show the company are major players in well-established industries and likely to be a steady company. In another side, DER (Debt to Equity Ratio) indicate the relative proportion of shareholders equity and debt used to finance company's assets. More specifically, it reflects the ability of shareholders equity to cover all outstanding debts in the event of a business downtrum

Using these conditions, the chosen company that fits the standards are:

Table 1: Market Cap. And DER value of the chosen companies

\begin{tabular}{lllll}
\hline No & $\begin{array}{l}\text { Stocks } \\
\text { Code }\end{array}$ & Company Name & Market Cap. Value & DER \\
\hline 1 & ASII & Astra International Tbk. & 289.457 .404 .951 .000 & 0,91 \\
2 & INKP & Indah Kiat Pulp \& Paper Tbk. & 105.042 .872 .467 .200 & 1,35 \\
3 & TLKM & Telekomunikasi Indonesia Tbk. & 359.855 .987 .148 .000 & 1,05 \\
4 & UNTR & United Tractors Tbk. & 131.487 .263 .544 .000 & 0,72 \\
5 & UNVR & Unilever Indonesia Tbk. & 329.997 .500 .000 .000 & 0,76 \\
\hline
\end{tabular}


The first step of analysis process was done by calculating the value of return, expected return and risk (variance), and covariance. The result of expected return, risk, and covariance of each products are:

Table 2: Expected retuned and Risk value of the chosen companies

\begin{tabular}{cccc}
\hline No & Stocks Code & Expected Return & Risk \\
\hline 1 & ASII & 0.00424 & 0.00424 \\
2 & INKP & 0.06401 & 0.0279 \\
3 & TLKM & 0.01038 & 000357 \\
4 & UNTR & 0.01412 & 0.00546 \\
5 & UNVR & 0.009201 & 0.00273 \\
\hline
\end{tabular}

Table 3: Covariance value of the chosen companies

\begin{tabular}{cccccc}
\hline $\begin{array}{c}\text { Stocks } \\
\text { Code }\end{array}$ & ASII & INKP & TLKM & UNTR & UNVR \\
\hline ASII & 0.00424 & 0.000475 & 0.00114 & 0.00157 & 0.00074 \\
INKP & 0.00475 & 0.0279 & -0.00276 & 0.00333 & -0.000377 \\
TLKM & 0.00114 & -0.00276 & 0.00357 & -0.00032 & 0.00041 \\
UNTR & 0.00157 & 0.00333 & -0.00032 & 0.00546 & -0.0000167 \\
UNVR & 0.00074 & -0.000377 & 0.00041 & -0.0000167 & 0.00273 \\
\hline
\end{tabular}

Based on Table 3, it is obtained that all variables have relationships, because there are no zero covariance's. ASII shares have a positive covariance value with four other shares, this indicates that ASII stocks and four other stocks move towards the same direction, or in other words if the ASII stock return value rises, the return value of the other four stocks will tend to rise, and vice versa. If two stocks have a negative covariance value, for example in INKP shares and TLKM shares, it indicates that both shares are moving in the opposite direction, or in other words if the INKP stock return value tends to rise, the TLKM return value will tend to decrease, and vice versa.

\subsection{Markowitz Model.}

The objective of minimizing value of risk with the formula of variance with two constrains written in formulas are:

$$
\text { Minimize } \sigma_{p}^{2}=\sum_{i=1}^{n} \sum_{j=1}^{n} w_{i} w_{j} \sigma_{i j}
$$


subject to:

$$
\begin{gathered}
\text { (1) } \sum_{i=1}^{n} w_{i}=1 \\
\text { (2) } E\left[R_{p}\right]=\sum_{i=1}^{n} w_{i} E\left(R_{i}\right)
\end{gathered}
$$

The first constrain indicates the sums up proportion of each stocks are one, and the second constrain indicate that expected return of portfolio are the multiply each proportion to its expected return of each stock. When working with portfolios, the use of matrix can greatly simplify many of the computations. The matrix form is:

$$
\begin{aligned}
& \text { Minimize } \sigma_{p}^{2}=\left(\begin{array}{c}
w_{1} \\
w_{2} \\
\vdots \\
w_{n}
\end{array}\right)^{T}\left(\begin{array}{cccc}
\sigma_{1}^{2} & \sigma_{12} & \cdots & \sigma_{1 n} \\
\sigma_{21} & \sigma_{2}^{2} & \cdots & \sigma_{2 n} \\
\vdots & \vdots & \ddots & \vdots \\
\sigma_{n 1} & \sigma_{n 2} & \cdots & \sigma_{n}^{2}
\end{array}\right)\left(\begin{array}{c}
w_{1} \\
w_{2} \\
\vdots \\
w_{n}
\end{array}\right) \\
& =\left(\begin{array}{cccc}
w_{1}^{2} \sigma_{1}^{2} & w_{1} w_{2} \sigma_{12} & \cdots & w_{1} w_{n} \sigma_{i n} \\
w_{2} w_{1} \sigma_{21} & w_{2}^{2} \sigma_{2}^{2} & \cdots & w_{2} w_{n} \sigma_{i n} \\
\vdots & \vdots & \ddots & \vdots \\
w_{n} w_{1} \sigma_{n 1} & w_{n} w_{2} \sigma_{n 2} & \cdots & w_{n}^{2} \sigma_{n}^{2}
\end{array}\right)=w^{T} V w \\
& \text { (1) }\left(\begin{array}{c}
w_{1} \\
w_{2} \\
\vdots \\
w_{n}
\end{array}\right)^{T}\left(\begin{array}{c}
1 \\
1 \\
\vdots \\
1
\end{array}\right)=w^{T} \mathbf{1}=1 \\
& \text { (2) }\left(\begin{array}{c}
w_{1} \\
w_{2} \\
\vdots \\
w_{4}
\end{array}\right)^{T}\left(\begin{array}{c}
\overline{r_{1}} \\
\overline{r_{2}} \\
\vdots \\
\overline{r_{n}}
\end{array}\right)=w^{T} E(R)=E[R p
\end{aligned}
$$

Based on the objective function and the constrains, the lagrange function is:

$$
L(w, \lambda, \theta)=w^{T} V w+\lambda\left(1-w^{T} \mathbf{1}\right)+\theta\left(E\left[R_{p}\right]-w^{T} E(R)\right)
$$

The optimal condition would be attained by the necessary condition:

$$
\begin{gathered}
\frac{\partial L}{\partial w}=2 V w-\lambda 1-\theta E(R)=0 \\
\frac{\partial L}{\partial \lambda}=w^{T} 1-1=0 \\
\frac{\partial L}{\partial \theta}=w^{T} E(R)-E\left[R_{p}\right]=0
\end{gathered}
$$


consider that formula (4) is non singular, then:

$$
w=V^{-1}\left(\frac{\lambda}{2} \mathbf{1}+\frac{\theta}{2} E(R)\right)
$$

Substitute formulas (5) and (6) to formula (7), then:

$$
a_{3} \frac{\lambda}{2}+a_{2} \frac{\theta}{2}=1 \text { and } a_{2} \frac{\lambda}{2}+a_{1} \frac{\theta}{2}=E\left[R_{p}\right]
$$

with : $a_{1}=\left(E(R)^{T} V^{-1} E(R)\right), a_{2}=\left(E(R)^{T} V^{-1} \mathbf{1}\right)$ and $a_{3}=\left(\mathbf{1}^{T} V^{-1} \mathbf{1}\right)$. If $\boldsymbol{\lambda}$ and $\theta$ was solved then the result is:

$$
\frac{\lambda}{2}=\frac{1}{D}\left(a_{1}-a_{2} E\left[R_{p}\right]\right) \text { and } \frac{\theta}{2}=\frac{1}{D}\left(-a_{2}+a_{3} E\left[R_{p}\right]\right)
$$

where $D=a_{1} a_{3}-a_{2}^{2}$. Subtitute the value of $\frac{\lambda}{2}$ and $\frac{\theta}{2}$ to formula (3) So it obtained:

$$
w=w_{0}\left(1-E\left[R_{p}\right]\right)+w_{1} E\left[R_{p}\right]
$$

where $w_{0}=\frac{1}{D}\left(a_{1} V^{-1} \mathbf{1}-a_{2} V^{-1} E(R)\right)$ and $w_{1}=\frac{1}{D}\left(a_{1} V^{-1} \mathbf{1}-a_{2} V^{-1} E(R)\right)+\frac{1}{D}\left(a_{3} V^{-1} E(R)-\right.$ $\left.a_{2} V^{-1} \mathbf{1}\right)$.

\section{Notation:}

Table 4: List of Notation

\begin{tabular}{cl}
\hline Notation & \multicolumn{1}{c}{ Explanation } \\
\hline$R_{i, t}$ & Stock Return $i$ period $t$ \\
$P_{i, t}$ & Stock price $i$ period $t$ \\
$P_{i, t-1}$ & Stock price $i$ period $t-1$ \\
$E(R)$ & Stock Expected return \\
$w_{i}$ & Stock proportion $i$ \\
$E\left[R_{p}\right]$ & Portfolio expected return \\
$E\left(R_{i}\right)$ & Average price stock $i$ \\
$\sigma_{p}^{2}$ & Portfolio Variance return \\
$\sigma_{i j}$ & Covariance stock $i$ and $j$ \\
$V$ & Variance and Covariance matrix \\
$\mathbf{1}$ & Vector union dimension $a \times 1$ \\
\hline
\end{tabular}




\subsection{Stock Portfolio}

There are five selected companies which will then be diversified into one portfolio, which the five companies will be combined in a portfolio of two, three, four, and five combinations. The following is a portfolio combination formed:

Table 5: List of Portfolio

\begin{tabular}{|c|c|c|c|c|c|}
\hline $\mathrm{P}$ & Component & $\mathrm{P}$ & Component & $\mathrm{P}$ & Component \\
\hline$P_{1}$ & ASII, INKP & $P_{10}$ & UNTR, UNVR & $P_{19}$ & INKP, UNTR, UNVR \\
\hline$P_{2}$ & ASII, TLKM & $P_{11}$ & ASII, INKP, TLKM & $P_{20}$ & TLKM, UNTR, UNVR \\
\hline$P_{3}$ & ASII, UNTR & $P_{12}$ & ASII, INKP, UNTR & $P_{21}$ & ASII, INKP, TLKM, UNTR \\
\hline$P_{4}$ & ASII, UNVR & $P_{13}$ & ASII, INKP, UNVR & $P_{22}$ & ASII, INKP, TLKM, UNVR \\
\hline$P_{5}$ & INKP, TLKM & $P_{14}$ & ASII, TLKM, UNTR & $P_{23}$ & ASII, TLKM, UNTR, UNVR \\
\hline$P_{6}$ & INKP, UNTR & $P_{15}$ & ASII, TLKM, UNVR & $P_{24}$ & ASII, INKP, UNTR, UNVR \\
\hline$P_{7}$ & INKP, UNVR & $P_{16}$ & ASII, UNTR, UNVR & $P_{25}$ & INKP, TLKM, UNTR, UNVR \\
\hline$P_{8}$ & TLKM, UNTR & $P_{17}$ & INKP.TLKM, UNTR & $P_{26}$ & $\begin{array}{l}\text { ASII, INKP, TLKM, UNTR, } \\
\text { UNVR }\end{array}$ \\
\hline$P_{9}$ & TLKM, UNVR & $P_{18}$ & INKP.TLKM, UNVR & & \\
\hline
\end{tabular}

\subsection{Model Formulation}

The formulation of the Optimization Model of each portfolio is done with the aim of analyzing the portfolio to find out the optimal results of each portfolio, which will then be completed and reselected the optimal results from all portfolios, or the portfolio with the lowest risk.

Variables:

$$
\begin{aligned}
& w_{1}=\text { ASII proportion stock } \\
& w_{2}=\text { INKP proportion stock } \\
& w_{3}=\text { TLKM proportion stock } \\
& w_{4}=\text { UNTR proportion stock } \\
& w_{5}=\text { UNVR proportion stock }
\end{aligned}
$$

\begin{tabular}{|c|c|}
\hline Portfolio & Objective Function \\
\hline$P_{1}$ & $\begin{array}{l}\text { Minimize } \sigma_{P_{1}}^{2}\left(\mathrm{w}_{1}, \mathrm{w}_{2}\right)=0.00424 w_{1}^{2}+0.0279 w_{2}^{2}+0.000475 w_{1} w_{2} \\
\text { Subject to: } \\
\text { (1) } w_{1}+w_{2}=1 \\
\text { (2) } 0.00424 w_{1}+0.06401 w_{2}=0.05\end{array}$ \\
\hline$P_{2}$ & $\begin{array}{l}\text { Minimize } \sigma_{P_{2}}^{2}\left(w_{1}, w_{3}\right)=0.00424 w_{1}^{2}+0.00357 w_{3}^{2}+0.00114 w_{1} w_{3} \\
\text { Subject to: } \\
\text { (1) } w_{1}+w_{3}=1 \\
\text { (2) } 0.00424 w_{1}+0.01038 w_{3}=0.05\end{array}$ \\
\hline
\end{tabular}




\begin{tabular}{|c|c|}
\hline$P_{3}$ & $\begin{array}{l}\text { Minimize } \sigma_{P_{3}}^{2}\left(\mathrm{w}_{1}, \mathrm{w}_{4}\right)=0.00424 w_{1}^{2}+0.00546 w_{4}^{2}+0.001576 w_{1} w_{4} \\
\text { Subject to: } \\
\text { (1) } w_{1}+w_{4}=1 \\
\text { (2) } 0.00424 w_{1}+0.01412 w_{4}=0.05\end{array}$ \\
\hline$P_{4}$ & $\begin{array}{l}\text { Minimize } \sigma_{P_{4}}^{2}\left(\mathrm{w}_{1}, \mathrm{w}_{5}\right)=0.00424 w_{1}^{2}+0.00273 w_{5}^{2}+0.00074 w_{1} w_{5} \\
\text { Subject to: } \\
\text { (1) } w_{1}+w_{5}=1 \\
\text { (2) } 0.00424 w_{1}+0.0092 w_{5}=0.05\end{array}$ \\
\hline$P_{5}$ & $\begin{array}{l}\text { Minimize } \sigma_{P_{5}}^{2}\left(\mathrm{w}_{2}, \mathrm{w}_{3}\right)=0.0279 w_{2}^{2}+0.00357 w_{3}^{2}-0.00276 w_{2} w_{3} \\
\text { Subject to: } \\
\text { (1) } w_{2}+w_{3}=1 \\
\text { (2) } 0.06401 w_{2}+0.01038 w_{3}=0.05\end{array}$ \\
\hline$P_{6}$ & $\begin{array}{l}\text { Minimize } \sigma_{P_{6}}^{2}\left(\mathrm{w}_{2}, \mathrm{w}_{4}\right)=0.0279 w_{2}^{2}+0.0054 w_{4}^{2}+0.00333 w_{2} w_{4} \\
\text { Subject to: } \\
\text { (1) } w_{2}+w_{4}=1 \\
\text { (2) } 0.06401 w_{2}+0.01412 w_{4}=0.05\end{array}$ \\
\hline$P_{7}$ & $\begin{array}{l}\text { Minimize } \sigma_{P_{7}}^{2}\left(\mathrm{w}_{2}, \mathrm{w}_{5}\right)=0.0279 w_{2}^{2}+0.00273 w_{5}^{2}-0.00377 w_{2} w_{5} \\
\text { Subject to: } \\
\text { (1) } w_{2}+w_{5}=1 \\
\text { (2) } 0.06401 w_{2}+0.0092 w_{5}=0.05\end{array}$ \\
\hline$P_{8}$ & $\begin{array}{l}\text { Minimize } \sigma_{P_{8}}^{2}\left(\mathrm{w}_{3}, \mathrm{w}_{4}\right)=0.00357 w_{3}^{2}+0.00546 w_{4}^{2}-0.00032 w_{3} w_{4} \\
\text { Subject to: } \\
\text { (1) } w_{3}+w_{4}=1 \\
\text { (2) } 0.01038 w_{3}+0.01412 w_{4}=0.05\end{array}$ \\
\hline$P_{9}$ & $\begin{array}{l}\text { Minimize } \sigma_{P_{9}}^{2}\left(\mathrm{w}_{3}, \mathrm{w}_{5}\right)=0.00357 w_{3}^{2}+0.00273 w_{5}^{2}+0.00041 w_{3} w_{5} \\
\text { Subject to: } \\
\text { (1) } w_{3}+w_{5}=1 \\
\text { (2) } 0.01038 w_{3}+0.0092 w_{5}=0.05\end{array}$ \\
\hline$P_{10}$ & $\begin{array}{l}\text { Minimize } \\
\sigma_{P_{10}}^{2}\left(\mathrm{w}_{4}, \mathrm{w}_{5}\right)=0.00546 w_{4}^{2}+0.00273 w_{5}^{2}-0.0000167 w_{4} w_{5} \\
\text { Subject to: } \\
\text { (1) } w_{4}+w_{5}=1 \\
\text { (2) } 0.01412 w_{4}+0.010842 w_{5}=0.05\end{array}$ \\
\hline
\end{tabular}




\begin{tabular}{|c|c|}
\hline$P_{11}$ & $\begin{array}{l}\text { Minimize } \begin{array}{l}\sigma_{P_{11}}^{2}\left(\mathrm{w}_{1}, \mathrm{w}_{2}, \mathrm{w}_{3}\right)=0.00424 w_{1}^{2}+0.0279 w_{2}^{2}+0.00357 w_{3}^{2} \\
\quad+0.000475 w_{1} w_{2}+0.00114 w_{1} w_{3}-0.0027 w_{2} w_{3}\end{array} \\
\text { Subject to: } \\
\begin{array}{l}\text { (1) } w_{1}+w_{2}+w_{3}=1 \\
\text { (2) } 0.00424 w_{1}+0.06401 w_{2}+0.01038 w_{3}=0.05\end{array}\end{array}$ \\
\hline$P_{12}$ & $\begin{array}{l}\text { Minimize } \begin{array}{l}\sigma_{P_{12}}^{2}\left(\mathrm{w}_{1}, \mathrm{w}_{2}, \mathrm{w}_{4}\right)=0.00424 w_{1}^{2}+0.0279 w_{2}^{2}+0.00546 w_{3}^{2} \\
\quad+0.000475 w_{1} w_{2}+0.00157 w_{1} w_{4}+0.00333 w_{2} w_{4}\end{array} \\
\text { Subject to: } \\
\begin{array}{l}\text { (1) } w_{1}+w_{2}+w_{4}=1 \\
\text { (2) } 0.00424 w_{1}+0.06401 w_{2}+0.01412 w_{4}=0.05\end{array}\end{array}$ \\
\hline$P_{13}$ & $\begin{array}{l}\text { Minimize } \begin{array}{l}\sigma_{P_{13}}^{2}\left(\mathrm{w}_{1}, \mathrm{w}_{2}, \mathrm{w}_{5}\right)=0.00424 w_{1}^{2}+0.0279 w_{2}^{2}+0.00273 w_{5}^{2} \\
\quad+0.000475 w_{1} w_{2}+0.0074 w_{1} w_{5}-0.000377 w_{2} w_{5}\end{array} \\
\text { Subject to: } \\
\begin{array}{l}\text { (1) } w_{1}+w_{2}+w_{5}=1 \\
\text { (2) } 0.00424 w_{1}+0.06401 w_{2}+0.0042 w_{5}=0.05\end{array}\end{array}$ \\
\hline$P_{14}$ & $\begin{array}{l}\text { Minimize } \begin{array}{l}\sigma_{P_{14}}^{2}\left(\mathrm{w}_{1}, \mathrm{w}_{3}, \mathrm{w}_{4}\right)=0.00424 w_{1}^{2}+0.00357 w_{3}^{2}+0.00546 w_{4}^{2} \\
\quad+0.00114 w_{1} w_{3}+0.00157 w_{1} w_{4}+0.00032 w_{3} w_{4}\end{array} \\
\text { Subject to: } \\
\text { (1) } w_{1}+w_{3}+w_{4}=1 \\
\text { (2) } 0.00424 w_{1}+0.01038 w_{3}+0.01412 w_{4}=0.05\end{array}$ \\
\hline$P_{15}$ & $\begin{array}{l}\text { Minimize } \sigma_{P_{15}}^{2}\left(\mathrm{w}_{1}, \mathrm{w}_{3}, \mathrm{w}_{5}\right)=0.00424 w_{1}^{2}+0.00357 w_{3}^{2}+0.00273 w_{5}^{2} \\
\quad+0.00114 w_{1} w_{3}-0.000377 w_{1} w_{5}+0.00041 w_{3} w_{5} \\
\text { Subject to: } \\
\begin{array}{l}\text { (1) } w_{1}+w_{3}+w_{5}=1 \\
\text { (2) } 0.00424 w_{1}+0.01038 w_{3}+0.0092 w_{5}=0.05\end{array}\end{array}$ \\
\hline$P_{16}$ & $\begin{array}{l}\text { Minimize } \begin{array}{l}\sigma_{P_{16}}^{2}\left(\mathrm{w}_{1}, \mathrm{w}_{4}, \mathrm{w}_{5}\right)=0.00424 w_{1}^{2}+0.00546 w_{4}^{2}+0.00273 w_{5}^{2} \\
\quad+0.00157 w_{1} w_{4}+0.00074 w_{1} w_{5}-0.0000167 w_{4} w_{5}\end{array} \\
\text { Subject to: } \\
\begin{array}{l}\text { (1) } w_{1}+w_{4}+w_{5}=1 \\
\text { (2) } 0.00424 w_{1}+0.01412 w_{4}+0.0092 w_{5}=0.05\end{array}\end{array}$ \\
\hline$P_{17}$ & $\begin{array}{ll}\text { Minimize } & \sigma_{P_{17}}^{2}\left(\mathrm{w}_{2}, \mathrm{w}_{3}, \mathrm{w}_{4}\right)=0.0279 w_{2}^{2}+0.00357 w_{3}^{2}+0.00546 w_{4}^{2} \\
& -0.00276 w_{2} w_{3}+0.00333 w_{2} w_{4}-0.00032 w_{3} w_{4} \\
\text { Subject to: } & \end{array}$ \\
\hline
\end{tabular}




\begin{tabular}{|c|c|}
\hline & $\begin{array}{l}\text { (1) } w_{2}+w_{3}+w_{4}=1 \\
\text { (2) } 0.06401 w_{2}+0.01038 w_{3}+0.01412 w_{4}=0.05\end{array}$ \\
\hline$P_{18}$ & $\begin{array}{l}\text { Minimize } \sigma_{P_{18}}^{2}\left(\mathrm{w}_{2}, \mathrm{w}_{3}, \mathrm{w}_{5}\right)=0.0279 w_{2}^{2}+0.00357 w_{3}^{2}+0.00273 w_{5}^{2} \\
\quad-0.00276 w_{2} w_{3}-0.00377 w_{2} w_{5}+0.00041 w_{3} w_{5} \\
\text { Subject to: } \\
\text { (1) } w_{2}+w_{3}+w_{5}=1 \\
\text { (2) } 0.06401 w_{2}+0.01038 w_{3}+0.0092 w_{5}=0.05\end{array}$ \\
\hline$P_{19}$ & 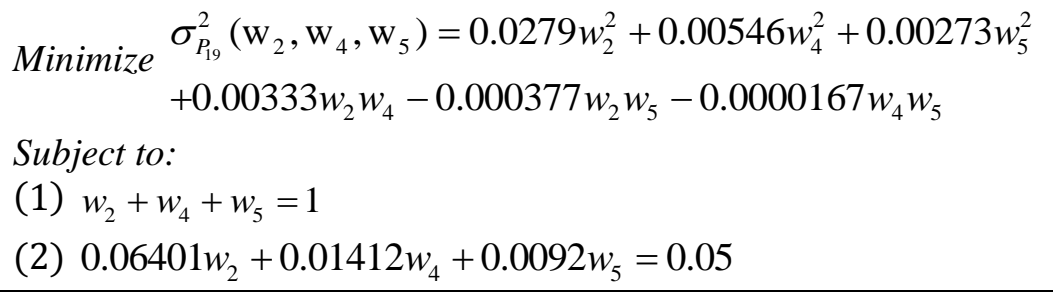 \\
\hline$P_{20}$ & $\begin{array}{l}\text { Minimize } \sigma_{P_{20}}^{2}\left(\mathrm{w}_{3}, \mathrm{w}_{4}, \mathrm{w}_{5}\right)=0.00357 w_{3}^{2}+0.00546 w_{4}^{2}+0.00273 w_{5}^{2} \\
\quad-0.00032 w_{3} w_{4}+0.00041 w_{3} w_{5}-0.0000167 w_{4} w_{5} \\
\text { Subject to: } \\
\text { (1) } w_{3}+w_{4}+w_{5}=1 \\
\text { (2) } 0.01038 w_{3}+0.01412 w_{4}+0.0092 w_{5}=0.05\end{array}$ \\
\hline$P_{21}$ & $\begin{array}{l}\text { Minimize } \\
\sigma_{P_{21}}^{2}\left(\mathrm{w}_{1}, \mathrm{w}_{2}, \mathrm{w}_{3}, \mathrm{w}_{4}\right)=0.00424 w_{1}^{2}+0.0279 w_{2}^{2}+0.00357 w_{3}^{2} \\
+0.00546 w_{4}^{2}+0.000475 w_{1} w_{2}+0.00114 w_{1} w_{3}+0.00157 w_{1} w_{4} \\
-0.0027 w_{2} w_{3}+0.00333 w_{2} w_{4}-0.0032 w_{3} w_{4} \\
\text { Subject to: } \\
\text { (1) } w_{1}+w_{2}+w_{3}+w_{4}=1 \\
\text { (2) } 0.00424 w_{1}+0.06401 w_{2}+0.01038 w_{3}+0.01412 w_{4}=0.05\end{array}$ \\
\hline$P_{22}$ & $\begin{array}{l}\text { Minimize } \\
\sigma_{P_{22}}^{2}\left(\mathrm{w}_{1}, \mathrm{w}_{2}, \mathrm{w}_{3}, \mathrm{w}_{5}\right)=0.00424 w_{1}^{2}+0.0279 w_{2}^{2}+0.00357 w_{3}^{2} \\
+0.00273 w_{5}^{2}+0.000475 w_{1} w_{2}+0.00114 w_{1} w_{3}+0.00074 w_{1} w_{5} \\
-0.00276 w_{2} w_{3}-0.00377 w_{2} w_{5}+0.00041 w_{3} w_{5} \\
\text { Subject to: } \\
\text { (1) } w_{1}+w_{2}+w_{3}+w_{5}=1 \\
\text { (2) } 0.00424 w_{1}+0.06401 w_{2}+0.01038 w_{3}+0.009201 w_{5}=0.05\end{array}$ \\
\hline$P_{2}$ & Minimize \\
\hline
\end{tabular}




\begin{tabular}{|c|c|}
\hline & $\begin{array}{l}\sigma_{P_{23}}^{2}\left(\mathrm{w}_{1}, \mathrm{w}_{3}, \mathrm{w}_{4}, \mathrm{w}_{5}\right)=0.00424 w_{1}^{2}+0.00357 w_{3}^{2}+0.00546 w_{4}^{2} \\
+0.00273 w_{5}^{2}+0.00114 w_{1} w_{3}+0.00157 w_{1} w_{4}+0.00074 w_{1} w_{5} \\
-0.00032 w_{3} w_{4}+0.00041 w_{3} w_{5}-0.000167 w_{4} w_{5} \\
\text { Subject to: } \\
\text { (1) } w_{1}+w_{3}+w_{4}+w_{5}=1 \\
\text { (2) } 0.00424 w_{1}+0.01038 w_{3}+0.01412 w_{4}+0.009201 w_{5}=0.05\end{array}$ \\
\hline$P_{24}$ & $\begin{array}{l}\text { Minimize } \\
\sigma_{P_{24}}^{2}\left(\mathrm{w}_{1}, \mathrm{w}_{2}, \mathrm{w}_{4}, \mathrm{w}_{5}\right)=0.00424 w_{1}^{2}+0.0279 w_{2}^{2}+0.00546 w_{4}^{2} \\
+0.00273 w_{5}^{2}+0.000475 w_{1} w_{2}+0.00157 w_{1} w_{4}+0.00074 w_{1} w_{5} \\
+0.00333 w_{2} w_{4}-0.0000377 w_{2} w_{5}-0.000167 w_{4} w_{5} \\
\text { (1) } w_{1}+w_{2}+w_{4}+w_{5}=1 \\
\text { (2) } 0.00424 w_{1}+0.06401 w_{2}+0.01412 w_{4}+0.009201 w_{5}=0.05\end{array}$ \\
\hline$P_{25}$ & $\begin{array}{l}\text { Minimize } \\
\sigma_{P_{25}}^{2}\left(\mathrm{w}_{2}, \mathrm{w}_{3}, \mathrm{w}_{4}, \mathrm{w}_{5}\right)=0.0279 w_{2}^{2}+0.00357 w_{3}^{2}+0.00546 w_{4}^{2} \\
+0.00273 w_{5}^{2}-0.00276 w_{2} w_{3}+0.00333 w_{2} w_{4}-0.000377 w_{2} w_{5} \\
-0.00032 w_{3} w_{4}+0.00041 w_{3} w_{5}-0.000167 w_{4} w_{5} \\
\text { Subject to: } \\
\text { (1) } w_{2}+w_{3}+w_{4}+w_{5}=1 \\
\text { (2) } 0.06401 w_{2}+0.01038 w_{3}+0.01412 w_{4}+0.009201 w_{5}=0.05\end{array}$ \\
\hline$P_{26}$ & $\begin{array}{l}\text { Minimize } \\
\sigma_{P_{26}}^{2}\left(x_{1}, x_{2}, x_{3}, x_{4}, x_{5}\right)=0.00424 w_{1}^{2}+0.0279 w_{2}^{2}+0.00357 w_{3}^{2} \\
+0.00546 w_{4}^{2}+0.00273 w_{5}^{2}+0.000475 w_{1} w_{2}+0.00114 w_{1} w_{3} \\
+0.00157 w_{1} w_{4}+0.00075 w_{1} w_{5}-0.0027 w_{2} w_{3}+0.00333 w_{2} w_{4} \\
-0.000377 w_{2} w_{5}-0.0032 w_{3} w_{4}-0.0000167 w_{4} w_{5} \\
\text { Subject to: } \\
\text { (1) } w_{1}+w_{2}+w_{3}+w_{4}+w_{5}=1 \\
0.00424 w_{1}+0.06401 w_{2}+0.01038 w_{3}+0.01412 w_{4} \\
\text { (2) }+0.009201 w_{5}=0.05\end{array}$ \\
\hline
\end{tabular}

\section{Result Analysis}

Based on the result of the 26 portfolios analyzed, there were several portfolios which did not reach return expectations of $5 \%$, namely $P_{2}, P_{3}, P_{4}, P_{8}, P_{9}, P_{10}, P_{14}, P_{15}, P_{16}, P_{20}$, and $P_{23}, P_{11}$ portfolios were not taken into consideration in portfolio selection optimal. Portfolios that provide 
the minimum risk and fulfill assumptions The return expectation of 5\% is $P_{26}$ consisting of five shares (ASII, INKP, TLKM, UNTR, UNVR), with the proportion of INKP shares 55.96\%, TLKM $33.16 \%$, UNTR $10.88 \%$ and the rest are $0 \% . P_{26}$ produces the lowest risk of $0.886 \%$. This investment proportion states the amount of money investors must invest to get minimum risk. Based on 26 stock portfolios analyzed, $P_{26}$ is the portfolio with the most share combinations from other portfolios, and produces the lowest risk. So, the more variables or stocks considered in one portfolio, the portfolio will provide more optimal results.

\section{Conclusion}

Based on the results of the research that has been carried out, several things can be concluded. First, more portfolios with members of the stock will result in lower risks compared to portfolios with fewer members, thus providing optimal diversification solutions. Second, after five companies were selected, 26 portfolios where Portfolio 26 (P_26) could be formed with ASII, INKP, TLKM, UNTR, and UNVR stock members were the optimal portfolio combination of all shares diversification. Third, the proportion of each share from P_26 is INKP shares 55.96\%, TLKM $33.16 \%$, UNTD $10.88 \%$, ASII $0 \%$, and UNVR $0 \%$, with the lowest risk of $0.886 \%$.

\section{References}

Kalfin, Sukono and Carnia, E. (2019). Optimization of the mean-absolute deviation portfolio investment in some mining stocks using the singular covariance matrix method. IOP Conf. Series: Journal of Physics: Conf. Series, 1315 (2019) 012002

Kalfin, Sukono and Carnia, E. (2019). Portfolio Optimization of the Mean-Absolute Deviation Model of Some Stocks using the Singular Covariance Matrix. International Journal of Recent Technology and Engineering (IJRTE), ISSN: 2277-3878, Volume-8, Issue-3.

Cohen, K. J. and Jerry, A. P. 1967. An Empirical Evaluation Portfolio-Selection Models. The Journal of Bussiness, University of Chicago, Vol 40, 166.

Edwin, J. P. and Ridgon. 2010. Kalkulus. Erlangga: Jakarta.

Husnan, S. 2005. Dasar-Dasar Teori Portofolio dan Analisis Sekuritas. Vol 5, Yogyakarta : UPP AMP YKPN

Jensen, M. C. 1969. The Performance of Mutual Funds in the Period 1945- 1964. Journal of Finance, V. 23, No. 2: pp 389-416.

Kazan, H. dan K. Uludag. 2014. Credit Portfolio Selection According to Sectors In Risky Environments: Markowitz Practice. Asian Economic and Financial Review, Vol. 4, No. 9: 1208-1219.

Marling, H. dan Emanuelsson ,S.. 2012. The Markowitz Portfolio Theory.

Rao, S. S. 2009. Engineering Optimization Theory and Practice. Fourth Edition. John Wiley \& Sons, Inc., Hoboken, New Jersey. 
Tandelilin, E. 2010. Analisis Investasi dan Manajemen Portofolio. Yogyakarta : BPFE 